PSYCHOMETRIKA-VOL. 2 , No. 3

SEPTEMBER, 1937

\title{
THE DETERMINATION OF THE FACTOR LOADINGS OF A GIVEN TEST FROM THE KNOWN FACTOR LOADINGS OF OTHER TESTS
}

\author{
PAUL S. DWYER \\ University of Michigan
}

\begin{abstract}
A technique is indicated by which approximations to the factor loadings of a new test may be obtained if factor loadings of a given group of tests and the correlations of the new test with the other tests are known. The technique is applicable to any orthogonal system and is especially adapted to cases in which $\Sigma a_{j i} a_{j k}=0$ when $i \neq k$. Application is also made to the simultaneous determination of the factor weights of a group of tests in which no additional common factor is present. The technique is useful in adding tests to a completed factorial solution and in using factorial solutions involving errors to give results which are approximately correct.
\end{abstract}

It happens not infrequently that, after a set of intercorrelations has been subjected to a multiple factor analysis, additional correlar tions with other tests are available. One naturally wishes to enlarge his analysis to include this new material. The technique explained below is devoted to the development of a means of incorporating the new results without the disheartening necessity of repeating the whole factorial solution.

Suppose that the intercorrelations of tests $1,2, \cdots j, \cdots, r$ are subjected to a multiple factor analysis which results in $k$ common factors. The resulting weights of each of the $r$ tests, and the communality of each, are indicated in Table $\mathrm{I}$,

TABLE I

\begin{tabular}{c|c|c|c|c|c}
\hline Test & $a_{j 1}$ & $a_{j 2}$ & $\cdots$ & $a_{j k}$ & $h_{j}{ }^{2}$ \\
\hline 1 & $a_{11}$ & $a_{12}$ & $\cdots$ & $a_{1 k}$ & $h_{1}{ }^{2}$ \\
\hline 2 & $a_{21}$ & $a_{22}$ & $\cdots$ & $a_{2 k}$ & $h_{2}{ }^{2}$ \\
\hline 3 & $a_{31}$ & $a_{32}$ & $\cdots$ & $a_{3 k}$ & $h_{3}{ }^{2}$ \\
\hline$\cdots$ & $\cdots$ & $\cdots$ & $\cdots$ & $\cdots$ & $\cdots$ \\
\hline$j$ & $a_{j 1}$ & $a_{j 2}$ & $\cdots$ & $a_{j k}$ & $h_{j}{ }^{2}$ \\
\hline$\cdots$ & $\cdots$ & $\cdots$ & $\cdots$ & $\cdots$ & $\cdots$ \\
\hline$r$ & $a_{r 1}$ & $a_{r 2}$ & $\cdots$ & $a_{r k}$ & $h_{r}{ }^{2}$ \\
\hline \multicolumn{1}{|c}{} & $\cdots$ &
\end{tabular}


where the $k$ reference vectors constitute any orthogonal system. Let $t$ be a test whose correlations with the $r$ tests are $r_{1 t}, r_{2 t}, \cdots, r_{r t}$ respectively.

To find the unknown reightings $a_{t 1}, a_{t 2}, \cdots a_{t k}$.

These weightings $a_{t i}$ must approximately satisfy the equations

$$
\begin{aligned}
& a_{t 1} a_{11}+a_{t 2} a_{12}+\cdots+a_{t i} a_{1 i}+\cdots+a_{t k} a_{1 k}=r_{1 t} \text {. } \\
& a_{t 1} a_{21}+a_{t 2} a_{22}+\cdots+a_{t i} a_{2 i}+\cdots+a_{t k} a_{2 k}=r_{2 t} \text {. } \\
& a_{t 1} a_{j 1}+a_{t 2} a_{j 2}+\cdots+a_{t i} a_{j i}+\cdots+a_{t k} a_{j k}=r_{j t} . \\
& a_{t 1} a_{r 1}+a_{t 2} a_{r 2}+\cdots+a_{t i} a_{r i}+\cdots+a_{t k} a_{r k}=r_{r t} .
\end{aligned}
$$

Applying the theory of least squares we get the normal equations

$$
\begin{aligned}
& a_{t 1} \Sigma a_{j 1}^{2}+a_{t 2} \Sigma a_{j_{1}} a_{j 2}+\cdots+a_{t i} \Sigma a_{j 1} a_{j i}+\cdots+a_{t k} \Sigma a_{j 1} a_{j k}=\Sigma a_{j 1} r_{j t} \text {, } \\
& a_{t 1} \Sigma a_{j 1} a_{j 2}+a_{t 2} \Sigma a_{j 2}^{2}+\cdots+a_{t i} \Sigma a_{j 2} a_{j i}+\cdots+a_{t k} \Sigma a_{j 2} a_{j k}=\Sigma a_{j 2} r_{j t} \text {, } \\
& a_{t 1} \Sigma a_{j 1} a_{j k}+a_{t 2} \Sigma a_{j 2} a_{j k}+\cdots+a_{t i} \Sigma a_{j 1} a_{j k}+\cdots+a_{t k} \Sigma a_{j k}^{2}=\Sigma a_{j k} r_{j t},
\end{aligned}
$$$$
\text { whose solution gives the desired } a_{t 1}, a_{t 2}, \cdots a_{t i}, \cdots a_{t k} \text {. }
$$

\begin{tabular}{|c|c|c|c|c|c|c|c|c|}
\hline & 1 & 2 & 3 & 4 & 5 & 6 & 7 & 8 \\
\hline 1 & $\ldots$ & .58 & -.28 & .01 & .36 & .38 & .61 & .15 \\
\hline 2 & .58 & $\ldots$ & -.28 & .10 & .52 & .44 & .78 & .10 \\
\hline 3 & -.28 & -.28 & $\ldots$ & .56 & .14 & -.56 & -.42 & -.70 \\
\hline 4 & .01 & .10 & .56 & $\ldots$ & .40 & -.34 & -.03 & -.65 \\
\hline 5 & .36 & .52 & .14 & .40 & $\ldots$ & .08 & .46 & -.30 \\
\hline 6 & .38 & .44 & -.56 & -.34 & .08 & $\ldots$ & .54 & .50 \\
\hline 7 & .61 & .78 & -.42 & -.03 & .46 & .54 & $\ldots$ & .25 \\
\hline 8 & .15 & .10 & -.70 & -.65 & -.30 & .50 & .25 & $\cdots$ \\
\hline
\end{tabular}$$
\text { The communality is } h_{t}^{2}=a_{t 1}{ }^{2}+a_{t 2}{ }^{2} \cdots+a_{t k}{ }^{2} \text {. }
$$

As an example consider a seven variable set of correlations used by Thurstone (1, page 129) together with the correlations of these seven variables with an eighth variable. The intercorrelations are presented in Table II.

TABLE II

The principal axes solution is given by Thurstone (1, page 128) and is repeated in Table III with the undetermined test 8 and the values of $r_{8 j}$ indicated. 
TABLE III

\begin{tabular}{c|c|c|c|c|c}
\hline Test & $r_{j \Lambda_{1}}$ & $r_{j \Lambda_{2}}$ & $r_{j \wedge_{3}}$ & $h^{2}$ & $r_{s j}$ \\
\hline 1 & +.659828 & +.120945 & .00 & .45 & .15 \\
2 & +.830332 & +.265611 & .00 & .76 & .10 \\
3 & -.541290 & +.637969 & .00 & .70 & -.70 \\
4 & -.126124 & +.770774 & .00 & .61 & -.65 \\
5 & +.437356 & +.590526 & .00 & .54 & -.30 \\
6 & +.637638 & -.336776 & .00 & .52 & .50 \\
7 & +.904489 & +.109084 & .00 & .83 & .25 \\
8 & $r_{8 \Lambda_{1}}$ & $r_{8 \Lambda_{3}}$ & .00 & $h_{8}{ }^{2}$ & \\
\hline
\end{tabular}

Thurstone shows $\sum_{j=1}^{7} r_{j}{ }^{2} \Lambda_{1}=2.849689, \sum_{j=1}^{7} r_{j}{ }^{2} \Lambda_{2}=1.560312$ and it is easily shown in addition that

$$
\sum_{j=1}^{7} r_{j \Lambda_{1}} r_{j \Lambda_{2}}=.000003, \sum_{j=1}^{7} r_{j \Lambda_{1}} r_{s j}=1.056625, \quad \sum_{j=1}^{7} r_{j \Lambda_{2}} r_{8 j}=-1.221153,
$$

so that the normal equations are

$$
\begin{aligned}
2.849689 r_{8 \Lambda_{1}}+.000003 r_{8 \Lambda_{2}} & =1.056625, \\
.000003 r_{8 \Lambda_{1}}+1.560312 r_{8 A_{2}} & =-1.221153,
\end{aligned}
$$

and approximately

with $h_{8}^{2}=.75$.

$$
\begin{aligned}
& r_{8 \Lambda_{2}}=\frac{1.056625}{2.849689}=.370786 \\
& r_{8 \Lambda_{2}}=\frac{-1.221153}{1.560312}=-.782634
\end{aligned}
$$

The solution of the normal equations is very simple when the non-diagonal coefficients in the normal equations are zero. This situation is attained in the illustration above and it appears (2, pages 425 426) in all cases in which a principal component solution is used. It is frequently approximately attained, when $k$ is large, when other orthogonal axes, centroid for example, are used. Thus the non-diagonal terms of Table $7(1$, page 131) are small when compared with the diagonal entries. Approximate values of weights for an additional test $t$ could be obtained by treating each non-diagonal entry as 0 .

As a parenthetical remark we note that in case the non-diagonal entries are relatively small, they may be placed equal to zero. The diag- 
onal entries then give first approximations to roots of the characteristic equation. The actual two decimal place values of the roots of the characteristic equation of Table 7 (1, page 131) and the approximations as determined by inspection are

TABLE IV

\begin{tabular}{c|c}
\hline actual values & approximations \\
\hline-5.02 & -5.01 \\
-1.18 & -1.18 \\
-.44 & -.43 \\
-.32 & -.34 \\
\hline
\end{tabular}

Suppose that a system of three orthogonal reference vectors is used to summarize the correlations of Table I. Thurstone has used such a system (1, page 124). This table, augmented to include the indicated weights of test 8 and the correlation of test 8 with the other tests is given in Table $\mathrm{V}$.

TABLE V

\begin{tabular}{c|c|c|c|c|r}
\hline Tests & $a_{j 1}$ & $a_{j 2}$ & $a_{j 3}$ & $h_{j}^{2}$ & $r_{8 j}$ \\
\hline 1 & +.5 & -.2 & +.4 & .45 & .15 \\
2 & +.6 & -.2 & +.6 & .76 & .10 \\
3 & -.6 & +.5 & +.3 & .70 & -.70 \\
4 & -.3 & +.4 & +.6 & .61 & -.65 \\
5 & +.2 & +.1 & +.7 & .54 & -.30 \\
6 & +.6 & -.4 & 0 & .52 & .50 \\
7 & +.7 & -.3 & +.5 & .83 & .25 \\
8 & $a_{81}$ & $a_{82}$ & $a_{83}$ & $h_{8}^{2}$ & \\
\hline
\end{tabular}

From the principal axes solution it is expected that $h_{8}{ }^{2}=.75$.

It is desired to find $a_{81}, a_{82}, a_{83}$. The normal equations become

$$
\begin{aligned}
1.95 a_{81}-1.07 a_{82}+.69 a_{83} & =1.165, \\
-1.07 a_{81}+.75 a_{82}+.11 a_{83} & =-.965, \\
.69 a_{81}+.11 a_{82}+1.71 a_{83} & =-.565,
\end{aligned}
$$

where the coefficients on the left have been previously found by Thurstone (1, page 125) in computing the characteristic equation. The solution of these equations is the easily verified.

$$
\begin{aligned}
& a_{81}=.5, a_{82}=-.5, a_{83}=-.5 \text { with } \\
& a_{81}{ }^{2}+a_{82}{ }^{2}+a_{83}{ }^{2}=.75 \text { as expected. }
\end{aligned}
$$


A slight variation of the method makes possible the simultaneous computations of weightings on a number of tests. Denote the weightings on test $t$ by $a_{t 1}, \cdots, a_{t k}$ and the correlation of test $t$ with the other tests by $r_{t 1}, r_{t 2}, \cdots r_{t r}$.

Let

$$
R_{i t}=\sum_{j=1}^{r} a_{j i} r_{t j} \quad \text { and } \quad \sum_{j=1}^{r} a_{i_{j} j} a_{i_{2} j}=A_{i_{1} j_{2}} ;
$$

the normal equations are

$$
\begin{aligned}
& A_{11} a_{t 1}+A_{12} a_{t 2} \cdots+A_{1 j} a_{t j}+\cdots+A_{1 k} a_{t k}=R_{1 t}, \\
& A_{21} a_{t 1}+A_{22} a_{t 2} \cdots+A_{2 j} A_{t j}+\cdots+A_{2 k} a_{t k}=R_{2 t}, \\
& \cdot \cdot \cdot \cdot \cdot \cdot \cdot \cdot \cdot \cdot \cdot \cdot \cdot \cdot \cdot \cdot \cdot \cdot \cdot \cdot \\
& A_{k 1} a_{t 1}+A_{k 2} a_{t 2} \cdots+A_{k j} a_{t j}+\cdots+A_{k k} a_{t k}=R_{k t} .
\end{aligned}
$$

If $D$ is the determinant of the coefficients and $D_{i j}$ is the cofactor of $A_{i j}$, then

$$
a_{t j}=\frac{D_{1 j}}{D} R_{1 t}+\frac{D_{2 j}}{D} R_{2 t}+\cdots+\frac{D_{k j}}{D} R_{k t} .
$$

The determinantal coefficients are the same no matter what the value of $t$. After they are computed it is only necessary to insert the $R_{i t}$ to find the different weightings. This method is discussed in more detail in an article which will be published soon (3).

If a principal axes solution is found and another test is added to the solution by this method, the result will not give a principal axes solution of the new set of tests since the principal axes description of each test varies with the introduction or deletion of a test.

The general method outlined is also applicable when an error is found in one of the entries of the correlation matrix. For example, suppose that a mistake in sign was made in the value of $r_{i j}$ which was recorded as +.55 rather than -.55. A mistake of this kind is apt to be discovered as the centroid solution advances since this residual may not approach zero as do the others.

In lieu of a complete repetition of the analysis, the following method is indicated. Take the result of the analysis which used the erroneous $r_{i j}$ and cross out the weights for test $i$ and test $j$. The weights on the following tests give the desired correlations with all the tests except test $i$ and test $j$. Provide the weights for test $i$ and test $j$ by the method explained above using the correct correlations. The resulting centroid solution does not appear to give the same re- 
sult as a centroid solution using the correct intercorrelations, but, when rotated to reveal simple structure, the results are in approximate agreement if the residuals of test $i$ and $j$ are of the same order as the other residuals.

\section{REFERENCES}

1. Thurstone, L. L., The Vectors of Mind. University of Chicago Press, 1935.

2. Hotelling, H., "Analysis of a Complex of Statistical Variables into Principal Components," Journal of Educational Psychology, Sept.-Oct., 1933, 24.

3. DWYER, P. S., "The Simultaneous Computation of Groups of Regression Equations and Associated Multiple Correlation Coefficients,"Annals of Mathematical Statistics, December, 1937. 